\title{
Just the Facts: Extracorporeal cardiopulmonary resuscitation for out-of-hospital cardiac arrest
}

\author{
Shannon M. Fernando (D, MD, MSc ${ }^{*}$; Brian Grunau, MD, MHSc ${ }^{\ddagger \S}$; Daniel Brodie, MD\|ף \\ CLINICAL SCENARIO
}

A 58-year-old man is brought by the ambulance to the emergency department (ED) of a tertiary care centre following an out-of-hospital cardiac arrest. Paramedics were called by the patient's wife after he had collapsed. She immediately initiated cardiopulmonary resuscitation (CPR). Prior to his collapse, he had been complaining of chest pain. His initial rhythm in the field was ventricular fibrillation, and he received defibrillation. An automated CPR device was applied prior to transport. En route, return of spontaneous circulation is achieved. An electrocardiogram shows ST-segment elevation in the anterior leads. Just prior to arrival, the patient suffers recurrent cardiac arrest with two further rounds of unsuccessful defibrillation in the ED. At this point, a decision is made to proceed with extracorporeal cardiopulmonary resuscitation (ECPR), prior to transport for cardiac catheterization.

\section{KEY CLINICAL QUESTIONS}

\section{What is ECPR?}

ECPR is an extracorporeal life support strategy initiated during cardiac arrest, which serves to bypass the patient's heart and lungs (Figure 1). ${ }^{1}$ Venoarterial cannulation of the femoral artery and femoral vein are performed percutaneously or, if needed, by surgical cutdown. Blood is drained via the venous cannula and pumped through a membrane oxygenator for removal of carbon dioxide and addition of oxygen. Blood is then returned through the arterial cannula, typically at a speed of 3-5 L/min in order to maintain patient hemodynamics. ECPR for out-of-hospital cardiac arrest can be provided in the ED, in the cardiac catheterization laboratory, and even the prehospital setting, in systems set up to provide this resource. Initiation of ECPR in the ED should be made in conjunction with the extracorporeal life support team specialist, as the decision to proceed is predicated on the belief that there is a potentially reversible etiology of the cardiac arrest, or on the basis of candidacy for other forms of temporary or durable circulatory support (including heart transplant).

\section{Is ECPR beneficial in out-of-hospital cardiac arrest?}

There is conflicting data regarding the efficacy of ECPR. There are currently no available randomized trials evaluating ECPR in out-of-hospital cardiac arrest. Observational data provide little clarity on the ultimate efficacy of ECPR for out-of-hospital cardiac arrest. ${ }^{2}$ The incremental benefit of ECPR to emergency medical services (EMS) may vary depending on existing practices and outcomes, especially pertaining to intra-arrest transport. Results from studies

From the ${ }^{*}$ Department of Emergency Medicine, University of Ottawa, Ottawa, ON; ${ }^{\dagger}$ Division of Critical Care, Department of Medicine, University of Ottawa, Ottawa, ON; ${ }^{\ddagger}$ Department of Emergency Medicine, University of British Columbia, Vancouver, BC; ${ }^{\S}$ Centre for Health Evaluation and Outcome Sciences, Vancouver, BC; "Division of Pulmonary, Allergy, and Critical Care Medicine, Department of Medicine, Columbia University College of Physicians and Surgeons, New York, NY; and "Center for Acute Respiratory Failure, New York-Presbyterian Hospital, New York, NY.

Correspondence to: Dr. Shannon M. Fernando, Department of Critical Care, The Ottawa Hospital, 1053 Carling Ave., Ottawa, ON K1Y 4E9; Email: sfernando@qmed.ca.

(C) Canadian Association of Emergency Physicians 2020

CJEM 2020;22(6):760-763

DOI $10.1017 /$ cem.2020.450 


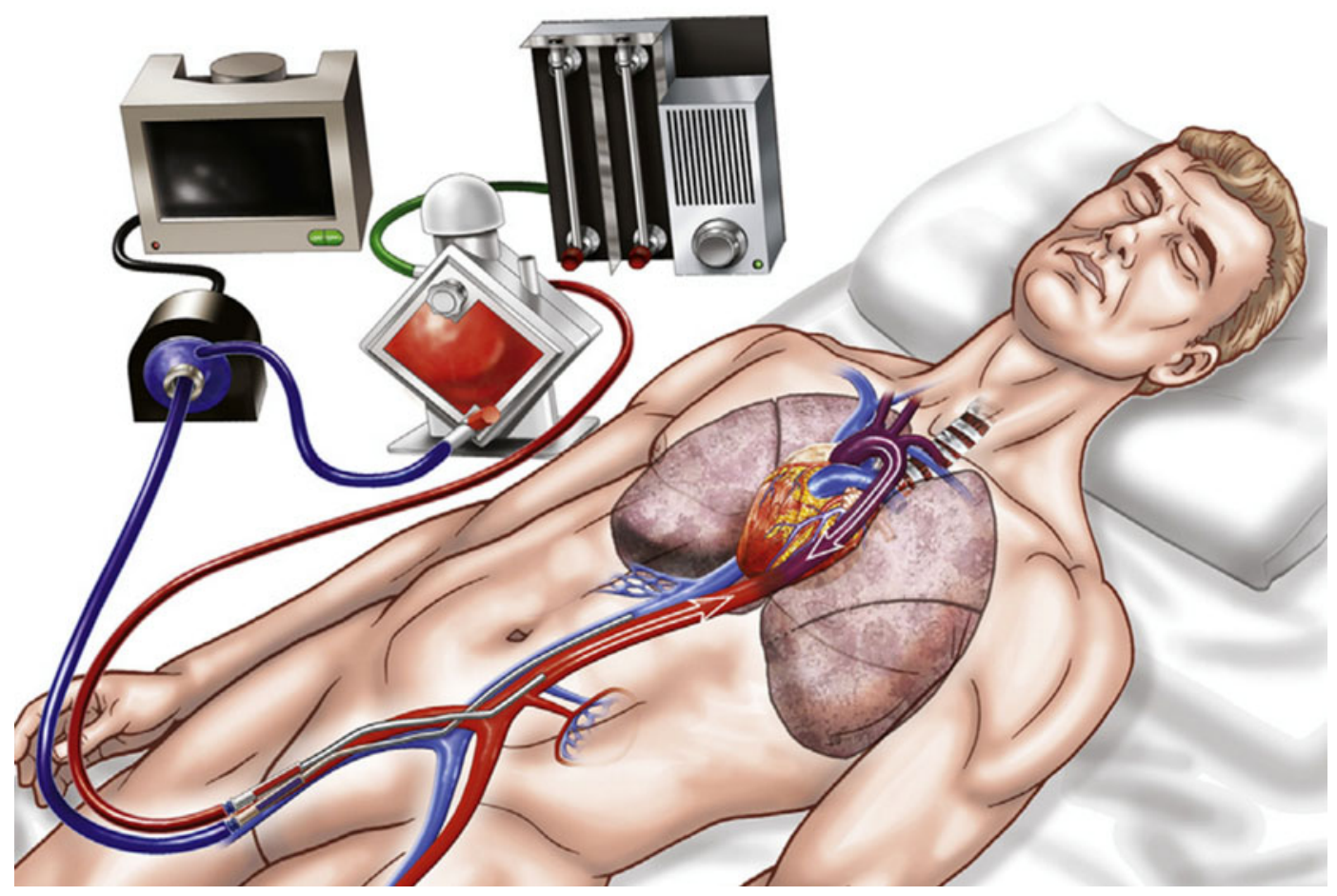

Figure 1. Depiction of a venoarterial extracorporeal membrane oxygenation circuit that could be used for extracorporeal cardiopulmonary resuscitation (ECPR). Deoxygenated blood (blue) is drained from the femoral vein, pumped through a membrane oxygenator where gas exchange takes place. Oxygenated blood (red) is then returned to the femoral artery and the systemic circulation. Image is adapted with permission from Abrams D, Combes A, Brodie D. Extracorporeal membrane oxygenation in cardiopulmonary disease in adults. J Am Coll Cardiol 2014;63:2769-78.

comparing ECPR with conventional resuscitation within EMS with comprehensive hospital transport policies are mixed, with some showing benefit. ${ }^{1,2}$ One high-volume ECPR program (using shockable initial cardiac rhythm as the primary indication for ECPR) reported a higher proportion of neurologically favourable survival than a comparable North American sample treated with conventional resuscitation (33\% v. $23 \%)$. ${ }^{3}$ Conversely, a population-based out-of-hospital cardiac arrest cohort study $(\mathrm{n}=13,191)$ comparing ECPR (a mix of prehospital and in-hospital initiation) with conventional resuscitation found no association with survival to discharge ( $8 \% \mathrm{v} .9 \%$, respectively), even after propensity matching. ${ }^{4}$ These observational studies are limited by selection bias with confounding by indication. Randomized trials are needed, especially in high-performing systems comparing on-scene resuscitation with intra-arrest transport for the purpose of ECPR initiation, and several are ongoing.

3.

What patients should be considered for ECPR, and what patients should not?

Several factors associated with prognosis following ECPR have been identified. Robust data to inform indications for ECPR are lacking; however, within ECPR-treated cohorts, factors associated with survival and favourable neurological status include initial shockable rhythm, witnessed arrest, bystander CPR, and transient return of spontaneous circulation prior to ECPR. ${ }^{3,4}$ The interval from cardiac arrest to ECPR initiation is highly associated with outcomes, with survival unlikely following intervals $>70$ minutes. Despite little supporting evidence, comorbidities should be considered in the eligibility assessment.

\section{What centres should consider developing an ECPR program?}

ECPR provision should be regionalized to specialized centres. The absolute number of out-of-hospital cardiac arrest lives saved from regional initiatives to improve the incidence and quality of bystander and EMS-provided 
resuscitation is likely to far exceed that of any ECPR program. Therefore, ECPR programs for out-of-hospital cardiac arrest should be considered in systems already demonstrating high overall positive outcomes. Further, given the resources required, a low proportion of expected successful outcomes and care complexity ECPR should be limited to centres with existing extracorporeal membrane oxygenation infrastructure and established multidisciplinary expertise in extracorporeal life support and cardiac care. ${ }^{5}$ Additionally, such centres should be capable of providing comprehensive post-arrest care (coronary angiography, therapeutic hypothermia). ED physicians play a critical role in identifying ECPR candidates in the ED or while still in the prehospital setting, activating ECPR initiation protocols, and participating within the ECPR initiation team.

\section{Are there any limitations or risks associated with ECPR?}

ECPR is associated with significant resource use and patient complications. There may be detrimental effects to the resuscitation quality of patients who are transported to the hospital for the purpose of ECPR initiation, which may be mitigated with training and careful protocol development. Patients treated with ECPR are at risk of major complications, including infection, thrombosis, bleeding, stroke, and limb ischemia, including amputation. Despite existing optimism regarding the use of ECPR, randomized data demonstrating efficacy are recommended prior to widespread implementation. The present evidence suggests that out-of-hospital cardiac arrest patients receiving ECPR likely still have high mortality (in the range of 70\%), despite the use of this treatment. ${ }^{3}$ ECPR may only demonstrate efficacy in particular subpopulations that are yet to be elucidated. This will ultimately have important implications for ED disposition, including the possibility of prehospital bypass of out-of-hospital cardiac arrest patients to centres capable of providing ECPR.

\section{CASE RESOLUTION}

The patient is cannulated for ECPR in the ED and transported for cardiac catheterization, which reveals a proximal lesion in the left anterior descending artery, and a stent is inserted. He returns to the Cardiac Intensive Care Unit where he is monitored for several days. Support is weaned to the point where he can be successfully decannulated and removed from ECPR. After several more weeks in hospital and rehabilitation with physiotherapy, he is ultimately discharged home independently.

\section{KEY POINTS}

- ECPR is a form of extracorporeal life support that is being increasingly used to provide artificial cardiac and respiratory support for selected patients with refractory out-of-hospital cardiac arrest.

- Existing observational data regarding the efficacy of ECPR is mixed and strongly confounded, highlighting the need for randomized data to support the use of this technology.

- Data supporting patient selection for ECPR is lacking, but patients should be considered on the basis of existing comorbidities, arrest characteristics (e.g. initial rhythm, witnessed), and the presumed likelihood of reversibility.

- ECPR is associated with important risks, including infection, bleeding, venous thromboembolism, and stroke.

- Given the above, ECPR should be provided at specialized centres capable of extracorporeal life support, postarrest care, and with access to other forms of mechanical circulatory support or heart transplant. 
Keywords: Extracorporeal life support, out of hospital cardiac arrest, cardiopulmonary resuscitation

Competing interests: BG received speaking honoraria from Stryker Corp. DB received research support from ALung Technologies, outside of the submitted work, and was previously on their medical advisory board. He has been on the medical advisory boards for Baxter, BREETHE, Xenios, and Hemovent.

\section{REFERENCES}

1. Grunau B, Hornby L, Singal RK, et al. Extracorporeal cardiopulmonary resuscitation for refractory out-of-hospital cardiac arrest: the state of the evidence and framework for application. Can 7 Cardiol 2018;34:146-55.

2. Beyea MM, Tillmann BW, Iansavichene AE, et al. Neurologic outcomes after extracorporeal membrane oxygenation assisted CPR for resuscitation of out-of-hospital cardiac arrest patients: a systematic review. Resuscitation 2018;130:146-58.

3. Bartos JA, Grunau B, Carlson C, et al. Improved survival with extracorporeal cardiopulmonary resuscitation despite progressive metabolic derangement associated with prolonged resuscitation. Circulation 2020;141:877-86.

4. Bougouin W, Dumas F, Lamhaut L, et al. Extracorporeal cardiopulmonary resuscitation in out-of-hospital cardiac arrest: a registry study. Eur Heart 7 2020;41:1961-71.

5. Abrams D, Garan AR, Abdelbary A, et al. Position paper for the organization of ECMO programs for cardiac failure in adults. Intensive Care Med 2018;44:717-29. 